\title{
CASOS DE ÉXITO DE EMPRENDIMIENTO SOCIAL EN GUAYAQUIL
}

\section{SUCCESSFUL SOCIAL ENTREPRENEURSHIP CASES IN GUAYAQUIL}

\section{Resumen:}

El presente trabajo introduce el concepto de emprendimiento social, y analiza la población beneficiada por los proyectos creados en Guayaquil por la Fundación Benéfica Acción Solidaria y Humane Escuela de Negocios. El objetivo principal de la investigación es demostrar la capacidad del modelo de emprendimiento social para convertirse en una forma de organización complementaria a la Economía Popular y Solidaria propuesta por el gobierno de Rafael Correa. Para cumplir dicho propósito, se utiliza un análisis descriptivo sobre los beneficios económicos y la correcta selección de la población favorecida en los proyectos de emprendimiento social de la Fundación y Humane. El estudio comparó los subempleados en Guayaquil con los trabajadores de los proyectos mencionados. La investigación revela que los trabajadores asociados a estos proyectos perciben en promedio ingresos mensuales superiores $y$ beneficios sociales adicionales a los trabajadores informales de la ciudad de Guayaquil.

Palabras Claves: emprendimiento Social, Economía Popular y Solidaria, mercado laboral, empleo informal, subempleo.

\section{INTRODUCCIÓN}

A diciembre de 2016, Ecuador presentaba un deterioro en el mercado laboral debido a la caída de la actividad económica, como consecuencia de la reducción en el precio del petróleo a partir de octubre de 2014 (Avellán, 2016). De acuerdo al Instituto Nacional de Estadísticas y Censos (INEC), la tasa de empleo adecuado a nivel nacional bajó desde $46,5 \%$ en diciembre de 2015 a 41,2\% en diciembre de 2016, lo que representó una pérdida de 243816 puestos de trabajos formales durante este periodo. De igual manera, el INEC reportó la tasa de subempleo más alta de la última década, la
Guillermo Avellán Solines gavellan@humane.edu.ec

Abstract:

This paper introduces the concept of social entrepreneurship and analyzes the population benefited by the projects created by Fundación Benéfica Acción Solidaria and Humane Business School. The main purpose of the research is to demonstrate the capacity of the Social Enterpreneurship Model to become a complementary form of organization to the Popular and Solidarity Economy proposed by the former President Rafael Correa. In order to achieve this goal, the paper introduces descriptive statistics on the economic benefits and the correct selection of the favored population in these social entrepreneurship projects. This study compared the underemployed people in Guayaquil with workers associated to the Social Entrepreneurship Model. This research reveals that workers within the model receive more social benefits and earn on average a monthly income greater than informal workers in Guayaquil.

Keywords: Social Entrepreneurship, Popular and Solidarity Economy, labor market, informal employment, underemployment.

cual aumentó desde $14 \%$ en 2015 , a $19,9 \%$ en diciembre de 2016. De esta manera, el número de personas subempleadas al cierre de 2016 se ubicó en 1564 825, registrando un aumento de 514179 personas respecto al año anterior (González, 2015). Es importante mencionar que las personas subempleadas son aquellas que trabajan menos de la jornada legal o perciben ingresos inferiores al salario mínimo, pero tienen deseo de trabajar horas adicionales. 
La reducción en el precio del crudo ecuatoriano provocó una disminución de la inversión pública del gobierno central. Por ejemplo, el gasto en capital disminuyó desde USD 11812,4 millones en diciembre de 2014, a USD 9 665,5 millones en diciembre de 2015. Ante la caída en el precio del petróleo, el Estado sufrió problemas de iliquidez en 2015 y 2016, que resultaron en atrasos de pagos a gobiernos autónomos descentralizados y proveedores de instituciones públicas. De acuerdo a publicaciones de diario El Comercio (2016), el gobierno central mantuvo impagos a pequeños negocios por varios meses, los cuales llegaron a realizar huelgas en el Ministerio de Finanzas para exigir sus desembolsos.

Para comprender cómo pequeños emprendimientos se convirtieron en contratistas del Estado, es necesario indicar que el gobierno del expresidente Rafael Correa impulsó el desarrollo de la pequeña y mediana empresa mediante la introducción de la Economía Popular y Solidaria (EPS). Según la Ley Orgánica de Economía Popular y Solidaria (LOEPS), se trata de una forma de organización económica donde sus integrantes, de manera individual o colectiva, se dedican a actividades comerciales o productivas basadas en relaciones de solidaridad, cooperación y reciprocidad, privilegiando al ser humano por sobre la apropiación, el lucro y la acumulación de capital, en armonía con la naturaleza (LOEPS, 2011). Así, el Estado promovió esta forma de organización a través de la aprobación de tres leyes: el Código Orgánico de la Producción, Comercio e Inversiones; la Ley Orgánica Reformatoria a la Ley Orgánica del Sistema Nacional de Contratación Pública; y la mencionada LOEPS. Estas leyes establecieron importantes incentivos para convertir a pequeños emprendimientos en proveedores de instituciones estatales a través de la contratación pública (Avellán, 2016).

Según cifras publicadas por el Instituto Nacional de Economía Popular y Solidaria (IEPS), la EPS ha registrado un crecimiento relevante mediante la contratación y créditos públicos. La Corporación Nacional de Finanzas Populares y Solidarias (Conafips) entregó, entre enero y noviembre de 2016, USD 100,05 millones a través de 55838 operaciones de crédito, con un promedio de USD 1792 por cada una. El gráfico 1 muestra cómo el monto en ventas de la EPS a instituciones públicas llegó a USD 83,52 millones en 2016, mientras que el monto, hacia los mercados privados, se ubicó en USD 12 millones en el mismo año. Además, esta política pública también repercutió de manera positiva en el empleo, como se puede apreciar en el gráfico 2. Las compras públicas generaron 10 445 plazas de trabajo; mientras que las compras del sector privado a la EPS crearon 2872 empleos (El Telégrafo, 2017).

\section{Gráfico 1: Ventas de la EPS por Sector}

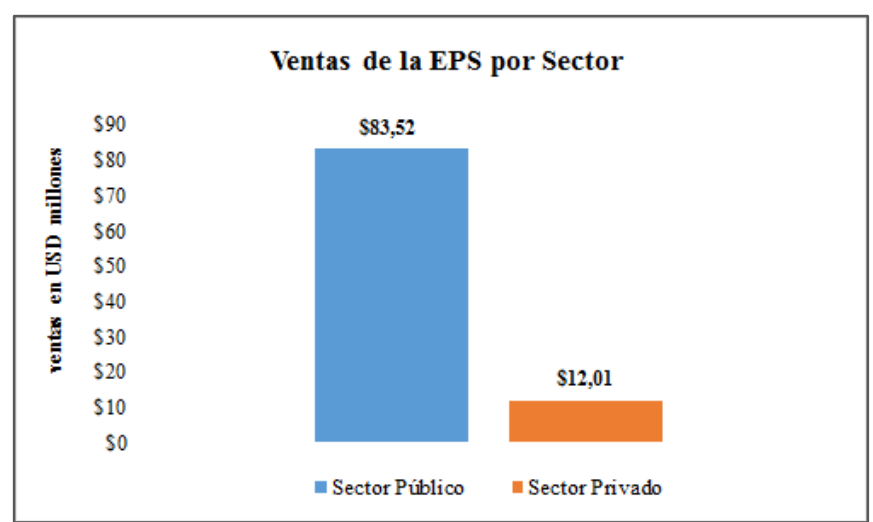

Fuente: El Telégrafo; Instituto Nacional de Economía Popular y Solidaria 


\section{Gráfico 2: Número de empleos generados por la EPS}

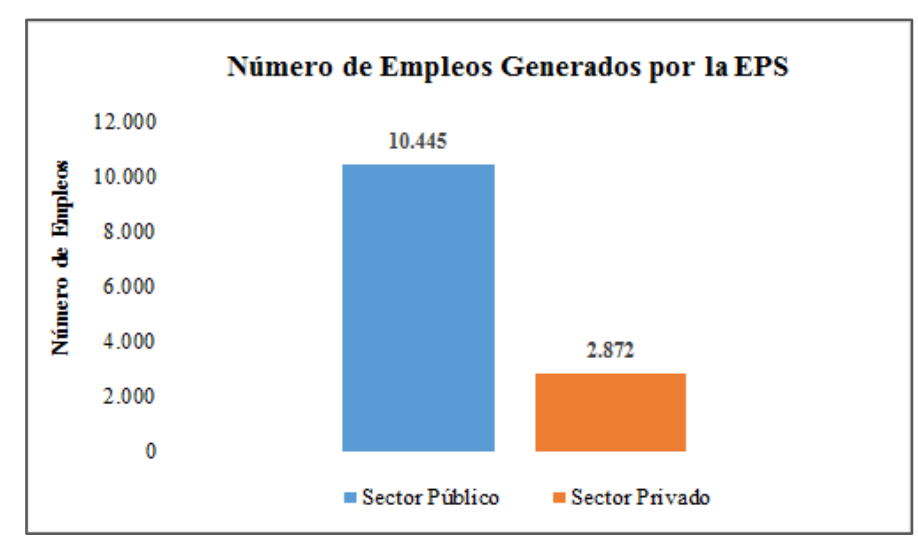

Fuente: El Telégrafo; Instituto Nacional de Economía Popular y Solidaria

También es relevante destacar las actividades con mayor participación en las ventas de la EPS al sector público en 2016. De acuerdo al IEPS, como se puede apreciar en el gráfico 3, el proyecto textil "Hilando el Desarrollo" posee la mayor participación de las ventas a instituciones públicas con el 52,4\%. El servicio de catering ocupa el segundo lugar con el 18,3\% de la participación en ventas. La manufactura textil tiene el 7,8\%, mientras que la metalmecánica ocupa el 5,2\% de las ventas al sector público.

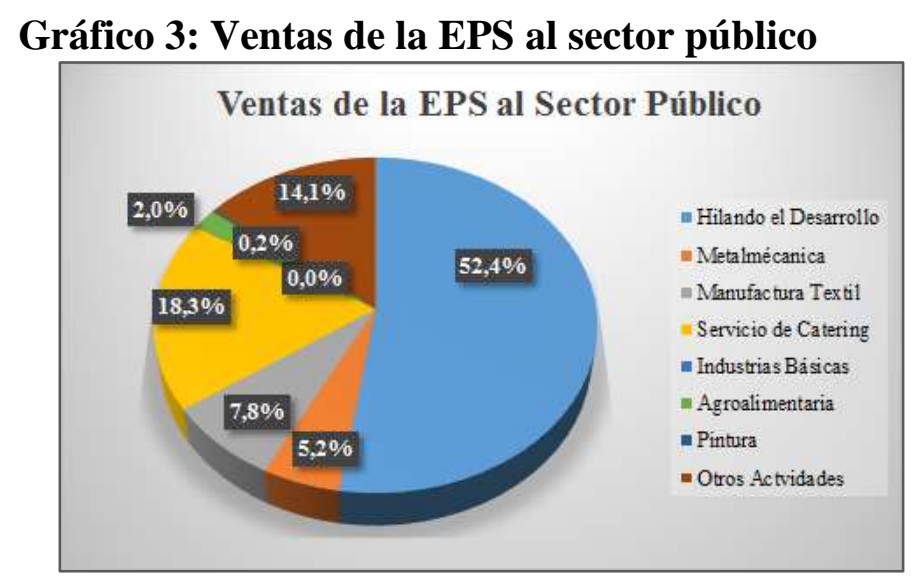

Fuente: El Telégrafo; Instituto Nacional de Economía Popular y Solidaria

Estas cifras reflejan la alta concentración de las ventas de la EPS hacia instituciones públicas. En el año 2016, el $87 \%$ de las ventas de la EPS estuvieron dirigidas al sector público, y tan solo el $13 \%$ fueron hacia el sector privado. De igual manera, las compras de instituciones públicas generaron el $78 \%$ de las plazas de trabajos en la EPS durante el 2016. Las compras del sector privado crearon el $22 \%$ de los empleos en el mismo periodo.

Este desbalance se convirtió en una dificultad para la EPS ante la caída en ingresos públicos, debido a la reducción del precio del petróleo y la disminución en la recaudación tributaria en 2016. Los problemas de iliquidez en el sector público han implicado menos contratos $\mathrm{y}$, por ende, menos ventas para la EPS. Esta dependencia exige pensar en un modelo complementario a la propuesta del gobierno basada principalmente en contratos públicos.

De esta manera, el Modelo Sostenible de Emprendimiento Social e Inclusión Laboral planteado por la Escuela de Negocios Humane y la Fundación Benéfica Acción Solidaria se convierte en una necesidad (Avellán, 2016). Este modelo ofrece nuevas oportunidades de negocio y crecimiento para los pequeños emprendimientos a través de alianzas estratégicas entre el sector público, el sector corporativo y la pequeña empresa. El objetivo 
central de esta propuesta es convertir a pequeños negocios y asociaciones en proveedores calificados de empresas privadas, a fin de reducir su dependencia de las compras de instituciones públicas.

En este sentido, Bargsted (2013) definió el emprendimiento social como una iniciativa de negocio que genera o no lucro, cuyo principal propósito es crear valor social. De igual manera, Muñiz (2015) indicó que la misión del emprendimiento social es brindar una solución a una problemática en particular, la cual no ha sido debidamente atendida por el mercado, ni por el Estado. De esta manera, la intención del emprendedor social es remediar, sin generar ganancias, problemas que impiden el desarrollo sostenible de los países. Esto coincide con Di Zhang y Swanson (2014), quienes concluyeron que los emprendimientos sociales son intrínsecamente sostenibles. Finalmente, basado en un estudio comparativo de diez emprendimientos sociales en México, Wulleman y Hudson (2015) encontraron que diferentes formas de emprendimiento han surgido de manera dinámica debido a los recursos y objetivos de los emprendedores sociales.

Respecto a la evidencia empírica, existen varios programas estatales de transferencias condicionadas en América Latina dirigidos a familias de escasos recursos, en donde las mujeres cumplen un rol fundamental en sus implementaciones. Estos programas sociales entregan transferencias monetarias a madres de familias muy pobres, bajo la condición que sus hijos cumplan revisiones médicas y asistan a centros educativos. Las evaluaciones de estos programas en la mayoría de países de la región han sido positivas en términos de salud, educación y consumo (Ibarrarán et al., 2017). Sin embargo, los casos de estudios no son tan frecuentes para iniciativas de emprendimiento social creadas por el sector privado y organizaciones sin fines de lucro. Una reciente investigación en Colombia de Acebedo y Velasco (2017) reveló la oportunidad para que la Universidad Autónoma de Bucaramanga ofrezca un diplomado en emprendimiento social dirigido a mujeres de escasos recursos, con el fin de combatir la pobreza y la exclusión de género. Asimismo, Acosta et al. (2018) establecieron la necesidad de desarrollar un programa formativo que fortalezca la cultura emprendedora de las mujeres en el Estado de Hidalgo en México, con los objetivos de generar empleo, reducir pobreza e inequidad y contribuir al desarrollo de la economía.

En la presente investigación, se analizan dos proyectos: primero, un taller de confección llamado Taller Narcisa de Jesús, que es proveedor calificado de Almacenes De Prati; el segundo, un proyecto denominado Casa del Lotero, donde vendedores informales se han convertido en vendedores capacitados de la Lotería Nacional. El taller fue creado y es administrado por la Fundación Benéfica Acción Solidaria; mientras que la Casa del Lotero está a cargo de la Escuela de Negocios Humane. El objetivo del presente estudio será demostrar los beneficios del Modelo de Emprendimiento Social como una forma de organización complementaria a la Economía Popular y Solidaria propuesta por el Gobierno de Rafael Correa. Se logrará dicho propósito mediante la presentación de estadísticas descriptivas sobre los beneficios económicos y la correcta selección de la población favorecida en los proyectos de emprendimiento social de la Fundación y Humane.

\section{Taller Narcisa de Jesús}

El Taller Narcisa de Jesús es un taller de confección de la Fundación Benéfica Acción Solidaria, que es proveedor calificado AAA de Almacenes De Prati. Se ha dedicado principalmente a la confección de blusas para la mujer contemporánea en tres marcas: $\mathrm{H} \& \mathrm{O}$, Isabella y Amanda, para las cuales produjo un total de 11000 prendas mensuales en promedio durante 2017.

El taller funciona desde el 2008. A inicios de 2017, trabajaban 47 personas en total, siendo la mayoría mujeres. Debido a la buena relación entre la Fundación y De Prati, se abrió una escuela de confección, llamada Taller de Mujeres Confeccionistas Emprendedoras, dirigida a mujeres de sectores vulnerables. Dicha escuela busca darles las herramientas y la 
formación necesarias para que sean mujeres emprendedoras.

\section{Casa del Lotero}

El proyecto la Casa del Lotero es un programa de responsabilidad social realizado por la Escuela de Negocios Humane para la Lotería Nacional y la Junta de Beneficencia de Guayaquil. Según un censo realizado por Humane, en 2016, el 50\% de la lotería se vende en Guayaquil y el $70 \%$ de los boletos son comercializados por vendedores informales. De esta manera, el proyecto busca responder a las necesidades de los vendedores de la Lotería Nacional y revalorizar su trabajo. El proyecto también tiene como propósito lograr la participación e integración de los vendedores de lotería y sus familias.

Este proyecto inició en el año 2011. Consigue sus objetivos a través de capacitaciones, asesoramiento, planes de salud y otras estrategias que permiten dignificar a los loteros y a sus actividades comerciales. Estos servicios tienen como propósito mejorar las condiciones de vida de los loteros, y fortalecer la imagen institucional de la Lotería Nacional.

La estructura de la investigación será la siguiente: la sección dos describe los datos y metodología utilizados en la investigación. La sección tres muestra los resultados y la discusión de los mismos. La sección cuatro menciona las implicaciones de política pública. Finalmente, la sección cinco contiene las conclusiones del trabajo.

\section{DATOS Y METODOLOGÍA}

El análisis comparativo utilizado en este estudio incluye las estadísticas de la Encuesta Nacional de Empleo, Desempleo y Subempleo publicada por el INEC a diciembre de 2016, específicamente la caracterización de las personas subempleadas en la ciudad de Guayaquil. También se utilizan datos mensuales correspondientes a los meses de septiembre y noviembre de 2016 de los proyectos de emprendimiento social como la Casa de Lotero de la Lotería Nacional y el Taller Narcisa de Jesús de Almacenes De Prati, respectivamente. Estos últimos fueron proporcionados por la Fundación Benéfica Acción Solidaria y la Escuela de Negocios Humane. Es importante mencionar que todas las cifras de las tres poblaciones analizadas provienen de un mismo periodo, por lo que son comparables en términos monetarios y de fechas.

La metodología aplicada en el presente trabajo es un análisis comparativo entre las características de las personas subempleadas en la ciudad de Guayaquil publicadas en la Encuesta de Empleo del INEC, y las estadísticas de los proyectos de emprendimiento social conocidos como: el Taller Narcisa de Jesús, administrado por la Fundación y la Casa del Lotero liderado por Humane. El objetivo de la investigación es demostrar la capacidad del Modelo de Emprendimiento Social como una forma de organización complementaria a la Economía Popular y Solidaria, a través de la presentación de estadísticas descriptivas sobre los beneficios salariales para la población favorecida en los proyectos de emprendimiento social de la Fundación y Humane.

Para demostrar la correcta selección de la población favorecida en los proyectos, se comparan las tres poblaciones antes mencionadas en las siguientes variables: sexo, edad, etnia, sectorización, tipo de empleo, seguro de salud, tiempo y experiencia laboral. Por otro lado, a fin de determinar los beneficios económicos para los colaboradores de estos proyectos, se evalúan los siguientes indicadores laborales: el ingreso mensual promedio, las horas promedio de trabajo a la semana y el ingreso promedio por hora.

Es importante explicar que se compara los proyectos de la Fundación Acción Solidaria y de Humane con las características principales de las personas subempleadas, ya que los colaboradores de estos proyectos en caso de no participar en estas iniciativas estarían en la informalidad. En el taller, la mayoría no contaba con empleo antes y, en el caso de la Casa del Lotero, estos siguen siendo informales.

\section{RESULTADOS Y DISCUSIÓN}


Tabla 1. Caracterización de subempleados en Guayaquil y empleados de taller de confección y loteros

\begin{tabular}{|c|c|c|c|c|}
\hline \multicolumn{2}{|c|}{ Característica } & $\begin{array}{l}\text { Subempleados } \\
\text { en Guayaquil }\end{array}$ & $\begin{array}{l}\text { Taller de } \\
\text { confección }\end{array}$ & Loteros \\
\hline \multirow{2}{*}{ Sexo } & Hombre & $58,6 \%$ & $13,6 \%$ & $52,0 \%$ \\
\hline & Mujer & $41,4 \%$ & $86,4 \%$ & $48,0 \%$ \\
\hline \multirow{5}{*}{ Edad } & Entre 15 y 24 años & $16,1 \%$ & $29,5 \%$ & $13,0 \%$ \\
\hline & Entre 25 y 34 años & $20,6 \%$ & $20,5 \%$ & $22,0 \%$ \\
\hline & Entre 35 y 44 años & $23,9 \%$ & $36,4 \%$ & $23,0 \%$ \\
\hline & Entre 45 y 64 años & $35,5 \%$ & $13,6 \%$ & $31,0 \%$ \\
\hline & Mayores a 65 años & $3,9 \%$ & $0,0 \%$ & $11,0 \%$ \\
\hline \multirow{6}{*}{ Etnia } & Indígena & $2,1 \%$ & & $54,0 \%$ \\
\hline & Afroecuatoriano & $1,9 \%$ & & $0,2 \%$ \\
\hline & Mestizo/a & $83,7 \%$ & $100,0 \%$ & $38,0 \%$ \\
\hline & Blanco & $2,3 \%$ & & $0,2 \%$ \\
\hline & Montubio & $4,3 \%$ & & $4,0 \%$ \\
\hline & Otro & $5,6 \%$ & & $3,6 \%$ \\
\hline \multirow{4}{*}{$\begin{array}{c}\text { Sectorización de la } \\
\text { Población con subempleo }\end{array}$} & Sector formal & $15,7 \%$ & $100,0 \%$ & \\
\hline & Sector informal & $75,3 \%$ & & $100,0 \%$ \\
\hline & Empleo Doméstico & $4,9 \%$ & & \\
\hline & No clasificados por sector & $4,1 \%$ & & \\
\hline \multirow{2}{*}{ Tipo de Empleado } & Empleado Público & $0,2 \%$ & & \\
\hline & Empleado Privado & $99,8 \%$ & $100,0 \%$ & $100,0 \%$ \\
\hline \multirow{3}{*}{ Seguro } & Seguro general & $10,9 \%$ & $100,0 \%$ & $3,0 \%$ \\
\hline & Otro seguro & $1,9 \%$ & & \\
\hline & Ninguno & $87,2 \%$ & & $97,0 \%$ \\
\hline \multirow{2}{*}{ Tipo de Trabajo } & Empleado Asalariado & $32,4 \%$ & $100,0 \%$ & \\
\hline & Empleado Independiente & $67,6 \%$ & & $100,0 \%$ \\
\hline \multirow{4}{*}{ Tiempo en este Trabajo } & Menos de 1 año & $21,4 \%$ & $13,6 \%$ & $1,0 \%$ \\
\hline & De 1 a 10 años & $54,8 \%$ & $84,1 \%$ & $31,0 \%$ \\
\hline & De 11 a 29 años & $17,3 \%$ & $2,3 \%$ & $51,0 \%$ \\
\hline & 30 y más & $6,6 \%$ & $0,0 \%$ & $17,0 \%$ \\
\hline \multirow{3}{*}{$\begin{array}{l}\text { Promedio Mensual del } \\
\text { Ingreso Laboral }^{(1)}\end{array}$} & Total & $\$ 217,93$ & $\$ 382,08$ & $\$ 277,50$ \\
\hline & Hombre & $\$ 248,00$ & $\$ 471,64$ & $\$ 300,00$ \\
\hline & Mujer & $\$ 173,00$ & $\$ 370,30$ & $\$ 255,00$ \\
\hline \multirow{3}{*}{$\begin{array}{c}\text { Horas Promedio de } \\
\text { Trabajo a la Semana } \\
\text { (Ocupación Principal y } \\
\text { Secundaria) } \\
\end{array}$} & Total & 27,18 & 50,00 & 41,53 \\
\hline & Hombre & 30,33 & 53,00 & 39,80 \\
\hline & Mujer & 22,72 & 47,00 & 43,25 \\
\hline \multirow{3}{*}{$\begin{array}{c}\text { Ingreso Promedio por } \\
\text { Hora }\end{array}$} & Total & $\$ 2,00$ & $\$ 1,91$ & $\$ 1,67$ \\
\hline & Hombre & $\$ 2,04$ & $\$ 2,22$ & $\$ 1,88$ \\
\hline & Mujer & $\$ 1,90$ & $\$ 1,97$ & $\$ 1,47$ \\
\hline
\end{tabular}

(1) No se considera el salario del bodeguero principal en el Taller de Confección, ni tampoco el subsidio diario de almuerzo por USD 0,55 en este proyecto. Pero sí se toman en cuenta las horas extras.

Fuente: INEC; Fundación Acción Solidaria y de Humane Escuela de Negocios.

El análisis comparativo entre las tres poblaciones analizadas en este estudio (subempleados de la ciudad de Guayaquil, colaboradores del Taller Narcisa de Jesús y vendedores de la Casa del Lotero) demuestran la correcta selección de los participantes de los 
proyectos de la Fundación y Humane. De acuerdo a la encuesta del INEC, el 58,6\% de las personas subempleadas fueron de sexo masculino, mientras que el $41,4 \%$ eran de sexo femenino. El Taller de Confección brinda una solución a las mujeres subempleadas ya que estas representaron el 86,4\% de los colaboradores del proyecto. Esto es importante porque las mujeres resultan más afectadas por el subempleo en términos de ingresos. Por su parte, la Casa del Lotero poseía una estructura por sexo bastante similar a las características de los subempleados en Guayaquil.

En relación a la edad, la población de subempleados en Guayaquil estaba distribuida, en su mayoría, entre personas de 25 y 64 años. El proyecto de la Casa del Lotero tenía proporciones parecidas en las tres principales categorías de edad de los subempleados de la urbe. Respecto a la etnia, los subempleados, en Guayaquil, se caracterizaron por ser mestizos $(83,7 \%)$, mientras que los colaboradores del Taller en su totalidad también eran mestizos. En la Casa del Lotero, los mestizos ocupaban el segundo puesto con un participación del 38\%. Los indígenas ocupan el primer puesto con una participación de $54 \%$, lo cual tiene un impacto positivo porque contribuye a dicho grupo, que generalmente reporta mayores tasas de pobreza y de necesidades insatisfechas (INEC, 2016).

Otro factor interesante es la composición de los subempleados por sectorización. El sector informal representó el 75,3\%, mientras que el sector formal llegó al 15,7\%. Los proyectos de la Fundación y Humane atienden a estos sectores: el taller se enfoca en brindar en su totalidad trabajo al sector formal y el proyecto con los loteros atiende en el $100 \%$ al sector informal. También es importante mencionar que ambos proyectos, al igual que el subempleo en Guayaquil, se caracterizan por estar compuestos en su mayoría por empleados del sector privado y no del sector público.

En variables relacionadas a la salud y seguridad social, las personas subempleadas que no poseían un seguro médico representan $87,2 \%$ del total. Afortunadamente, el Taller de Confección ofrece un contrato formal que incluye todos los beneficios de la ley, tal como estar afiliado al Instituto Ecuatoriano de
Seguridad Social (IESS). Sin embargo, el proyecto con los loteros no ofrecía hasta el 2017 un seguro médico a sus participantes, lo cual representará un reto y una oportunidad para los próximos años. No obstante, se ha venido realizando cada año entre 15 y 20 campañas de salud, en beneficio de los loteros.

En relación al tipo de trabajo, el empleado asalariado representaba el $32,4 \%$ y el empleado independiente correspondía al $67,6 \%$ de los subempleados en la ciudad de Guayaquil. Los proyectos tienen la particularidad de enfocarse en su totalidad en cada uno de estos tipos de trabajo. Por ejemplo, el $100 \%$ de las personas que trabajan en el taller son empleados asalariados, mientras que la totalidad de colaboradores que venden lotería son empleados independientes. De esta manera, se atiende los dos tipos de trabajo que tienen proporciones representativas de la población de subempleados.

En lo que respecta al tiempo en este trabajo, las personas subempleadas en Guayaquil, en su mayoría, se encontraba en la categoría entre 1 y 10 años de experiencia laboral. En el Taller de Confección, esta misma categoría de edad representó el 84,1\%; mientras que en la Casa del Lotero representó el $31 \%$ del total de colaboradores. En el proyecto de la Lotería, la categoría de experiencia con mayor incidencia estuvo entre 11 y 29 años realizando la misma actividad comercial.

Después de comparar estas variables entre las tres poblaciones analizadas en el presente estudio, se puede demostrar la correcta selección de las poblaciones favorecidas en los proyectos de la Fundación y Humane. Estas iniciativas de emprendimiento social buscan disminuir el número de subempleados, el cual alcanzó su nivel más alto de la última década a finales de 2016 e inicios de 2017.

Por otro lado, en términos económicos, los proyectos analizados permitieron a sus participantes (hombres y mujeres) percibir ingresos mensuales superiores a los recibidos por una persona subempleada en Guayaquil. En montos absolutos, el salario que percibieron las costureras del Taller Narcisa de Jesús fue de USD 370,30 sin considerar el subsidio diario de 
almuerzo por USD 0,55. Este salario fue superior a los USD 173,00 que recibió una mujer subempleada, y representó un incremento salarial de $114 \%$ respecto a una trabajadora subempleada. Además, los colaboradores del taller completaron la jornada legal y horas extras de trabajo, lo cual no es posible para una persona en el subempleo. Dado que existen diferencias en el número de horas trabajadas entre las personas subempleadas y los colaboradores de los proyectos, es necesario considerar el ingreso promedio por hora. Las costureras recibieron un salario de USD 1,97 por hora, el cual fue mayor que el USD 1,90 por hora que percibió una mujer subempleada.

En el mismo sentido, un hombre que participa en el proyecto la Casa del Lotero percibió un ingreso promedio de USD 300 al mes, lo cual significó un crecimiento de $21 \%$ en relación a un trabajador subempleado. Las mujeres que participaron de este proyecto recibieron un ingreso promedio de USD 255 al mes, que representó un incremento en ingresos de $47,40 \%$ en comparación con una mujer subempleada. Es importante precisar que prácticamente hombres y mujeres cumplen la jornada legal en este proyecto; sin embargo, sus ingresos fueron inferiores al salario básico unificado en 2016. No obstante, el ingreso promedio para una vendedora de lotería fue de USD 1,47 por hora, el cual fue inferior al que percibió una trabajadora subempleada que se ubicaba en USD 1,90 por hora. Del mismo modo, en el caso de los hombres, un lotero recibió USD 1,88 por hora, el cual fue inferior al USD 2,04 por hora para un trabajador subempleado. A pesar que los vendedores de lotería poseen un ingreso promedio por hora inferior en comparación con un trabajador en el subempleo en Guayaquil, es fundamental comprender que, en términos absolutos, el ingreso mensual promedio fue ampliamente superior para los empleados de la Casa del Lotero.

Finalmente, es relevante indicar las diferencias salariales entre hombres y mujeres para las tres poblaciones analizadas. En términos absolutos, un hombre en condición de subempleo ganó en promedio $43,4 \%$ más que una mujer. Del mismo modo, su ingreso por hora fue superior en 7,4\% al de una mujer. En taller de confección, los hombres en promedio percibieron un salario mayor al de las mujeres en $27,4 \%$, y su ingreso por hora también fue superior en $12,9 \%$. En el caso de la Casa del Lotero, el sueldo de las personas de sexo masculino fue mayor en $17,6 \%$ y su ingreso por hora también fue superior en $27,8 \%$.

\section{IMPLICACIONES}

La reducción en los ingresos públicos y los problemas de iliquidez del gobierno central ante el fin de la bonanza petrolera representaron una amenaza para la EPS, la cual tenía como socios estratégicos a varias instituciones públicas. Esta debilidad se evidencia ante la alta concentración de sus ventas y plazas de trabajo generadas por los contratos públicos. En el 2016, las compras del sector público representaron alrededor del $87 \%$ de las ventas totales de la EPS. Además, las compras públicas generaron aproximadamente el $78 \%$ de los empleos en este sector. La vulnerabilidad de los pequeños negocios ante una reducción en la contratación pública exige pensar en formas de organización alternativas a la EPS. De esta manera, el Modelo de Emprendimiento Social e Inclusión Laboral propuesto por la Escuela de Negocios Humane y la Fundación Acción Solidaria se convierte en una alternativa factible para brindar oportunidades de crecimiento y diversificación de ventas a los pequeños emprendimientos.

La importancia del Modelo de Emprendimiento Social Sostenible radica en incluir a pequeños negocios y asociaciones en cadenas de producción y comercialización, convirtiéndolos en proveedores o agentes de ventas calificados para diferentes productos y servicios. Otro factor relevante en este modelo es la sostenibilidad de las relaciones comerciales en el largo plazo, especialmente, al considerar los riesgos provocados por la incertidumbre de la contratación pública.

Para convertir en una realidad el Modelo de Emprendimiento Social Sostenible es necesario una alianza estratégica entre el sector público, organizaciones sin fines de lucro, el sector empresarial y los pequeños emprendimientos. Las organizaciones sin fines 
de lucro deben colaborar en la capacitación de la microempresa y servir como vínculo hacia nuevas relaciones comerciales con empresas a nivel nacional e internacional. Por su parte, el sector público debe promover facilidades para los pequeños y medianos emprendimientos como también establecer reconocimientos para empresas grandes, los cuales aumenten el interés y las posibilidades de establecer relaciones comerciales de largo plazo entre pequeños emprendimientos y el sector empresarial.

Respecto a los estímulos y reconocimientos otorgados por el sector público, los nuevos emprendimientos en el país deberían recibir capacitación técnica que facilite el acceso a distintas industrias. Por ejemplo, los organismos multilaterales podrían ofrecer asistencia técnica y líneas de crédito no reembolsables que financien la formación de capital humano y estudios de mercado para emprendimientos. Por otro lado, las compañías medianas y grandes podrían recibir reconocimientos o certificaciones por parte de instituciones públicas, como por ejemplo: certificados de comercio justo y de buenas prácticas empresariales. Estos reconocimientos tienen un alto valor en mercados internacionales como Europa, Estados Unidos y Asia. Sin embargo, tanto las facilidades como los reconocimientos deben ser aprobados a través de una legislación sencilla, objetiva y transparente, que evite cualquier tipo de simulación de empresas para acogerse a estos beneficios.

Por otro lado, es importante señalar que los proyectos de emprendimiento social analizados en esta investigación pueden servir como modelo para solucionar el subempleo en Guayaquil debido a dos razones: en primer lugar, la selección de la población beneficiada a través de estos proyectos posee un perfil similar a un trabajador informal de la ciudad. Adicionalmente, los proyectos permiten a su población beneficiada percibir salarios superiores en comparación a una persona subempleada. Además, los proyectos de emprendimiento social sostenible ofrecen varios beneficios adicionales, tales como seguro médico, campañas de salud, capacitaciones y subsidio de almuerzo.
De manera particular, el Taller Narcisa de Jesús representa una propuesta adecuada para disminuir el subempleo entre las mujeres a nivel nacional. Lo anterior significa la oportunidad de trabajar la jornada laboral completa y percibir al menos el salario básico unificado. De esta manera, el taller también se convierte en una herramienta fundamental para reducir la pobreza y transformar la vida de las personas más necesitadas.

Por su parte, la Casa del Lotero también permite a los vendedores de lotería recibir beneficios adicionales y percibir un ingreso mensual superior a las personas subempleadas. A pesar que perciben un ingreso por hora inferior, en relación a un trabajador informal, su ingreso mensual es superior en términos absolutos, lo cual tiene un impacto positivo para aquellas personas con varias necesidades insatisfechas.

Una limitación de la presente investigación es no incluir la variable de educación en el análisis comparativo debido a que el INEC no reporta el nivel de educación por categorías de empleo. Sin embargo, esta limitación parece denegarse cuando se compara la población subempleada de Guayaquil con los vendedores de lotería, que en su mayoría solo tiene educación primaria pero aun así perciben un salario mensual superior. De acuerdo a un censo realizado por Humane, el $62 \%$ de los vendedores de lotería solo completó la educación primaria; mientas que el 13\% no recibió educación formal. Adicionalmente, sería pertinente en próximas investigaciones poder validar los resultados estadísticos presentados en este artículo mediante métodos econométricos como evaluaciones de impacto, a fin de confirmar los beneficios de los proyectos analizados.

\section{CONCLUSIONES}

El Modelo de Emprendimiento Social Sostenible e Inclusión Laboral propuesto por Humane Escuela de Negocios y la Fundación Acción Solidaria representa una alternativa para la EPS implementada en el Gobierno de Rafael Correa. A través del análisis comparativo de las estadísticas correspondientes a las tres 
poblaciones estudiadas, se puede concluir que la selección de los colaboradores de los proyectos de la Fundación y Humane es correcta. Además, los trabajadores involucrados en estos proyectos perciben en promedio salarios superiores en comparación a las personas subempleadas en Guayaquil. Este modelo permite reducir la dependencia y vulnerabilidad que los pequeños negocios poseen ante la incertidumbre de la contratación pública. Esta iniciativa también es una excelente enseñanza para las microempresas interesadas en exportar sus productos y servicios a otros países, especialmente con la firma del Acuerdo Comercial con la Unión Europea.

\section{REFERENCIAS BIBLIOGRÁFICAS}

[1] Acebedo, M. y Velasco, M. (2017). Emprendimiento social femenino: prolegómenos conceptuales y estudio de casos. CIEG, 27:102-116.

[2] Acosta, S., Pedraza, E. y Sánchez, Y. (2018). Emprendimiento social femenino: una estrategia para el desarrollo. Boletín Científico INVESTIGIUM de la Escuela Superior de Tizayuca, 3 (6).

[3] Avellán, G. (2016). Efectos del Dólar y Petróleo en el Desempleo Urbano en Ecuador. Humane Escuela de Negocios.

[4] Bargsted, M. (2013). El emprendimiento social desde una mirada psicosocial. Civilizar, 13 (25): 121-132.

[5] Código Orgánico de la Producción, Comercio e Inversiones. Registro Oficial N. ${ }^{\circ} 351$, Suplemento. Quito, Ecuador, 29 de diciembre de 2010.

[6] Di Zhang, D., y Swanson, L. (2014). Linking social enterpreneurship and sustainability. Journal of Social Enterpreneurship, 5 (2): 175-191

[7] Diario El Comercio (2016). Proveedores del Estado impagos protestan en las afueras del Ministerio de Finanzas. Recuperado de http://www.elcomercio.com/actualidad/prov
Adicionalmente, los proyectos de emprendimiento social estudiados en esta investigación contribuirán a solucionar el subempleo en nuestro país, el cual es un problema estructural de la economía ecuatoriana. El mayor aporte del presente estudio fue demostrar que el segmento favorecido por estos proyectos posee características similares al de un trabajador subempleado en Guayaquil, especialmente, en la Casa del Lotero. Al mismo tiempo, se demostró que los participantes en estos proyectos perciben en promedio ingresos superiores y beneficios adicionales en comparación a las personas subempleadas en la urbe porteña

eedores-impagos-protestaministeriofinanzas.html

$\begin{array}{lrr}\quad[8] \quad E l \quad \text { Telégrafo } & (2017) . & 5.424 \\ \text { familias obtuvieron empleo } & \text { por } \\ \text { cofinanciamiento de iniciativas productivas. } & \\ \text { Recuperado re } & \text { de } \\ \text { http://www.eltelegrafo.com.ec/noticias/econ } \\ \text { omia/8/5-424-familias-obtuvieron-empleo- } \\ \text { por-cofinanciamiento-de-iniciativas- } \\ \text { productivas }\end{array}$

[9] Fundación Benéfica Acción Solidaria (2016). Estadísticas del Departamento Administrativo.

[10] González, M. (2015, octubre 29). La situación del empleo ha empeorado en el último año, contrario a lo que afirma el presidente. Recuperado de http://economiaenjeep.blogspot.com/2015/1 0/la-situacion-del-empleo-ha-empeoradoen.html

[11] Humane Escuela de Negocios (2016). Estadísticas del Departamento de Vinculación.

[12] Ibarrarán, P., Medellín, N., Regalia, F. y Stampini, M. (2017). How conditional cash transfers work: good practices after 20 years of implementation. Banco Interamericano de Desarrollo. Washington, D.C

[13] Instituto Nacional de Economía Popular y Solidaria. (2017). Rendición de 
www.economiasolidaria.gob.ec

[14] Instituto Nacional de Estadísticas y Censos (2016, diciembre). Encuesta Nacional de Empleo, Desempleo y Subempleo. Recuperado de http://www.ecuadorencifras.gob.ec/empleojunio-2017/

[15] Ley Orgánica de Economía Popular y Solidaria y del Sector Financiero Popular y Solidario. Registro Oficial N 444. Quito, Ecuador, 10 de mayo de 2011.

[16] Ley Orgánica Reformatoria a la Ley Orgánica del Sistema Nacional de Contratación Pública. Registro Oficial $\mathrm{N}^{\circ}$ 100, Segundo Suplemento. Quito, Ecuador, 14 de octubre de 2013.

[17] Muñiz, M. (2015). Monográfico Emprendimiento:innovación y cambio social. Revista Icade. Revista de las Facultades de Derecho y Ciencias Económicas y Empresariales, 0(94), 5-10. Recuperado de http://revistas.upcomillas.es/index.php/re vistaicade/article/view/5426/5242

[18] Wulleman, M. y Hudon, M. (2015). Models of social enterpreneurship: empirical evidence from Mexico. Journal of Social Enterpreneurship, 7 (2): 1-27. 